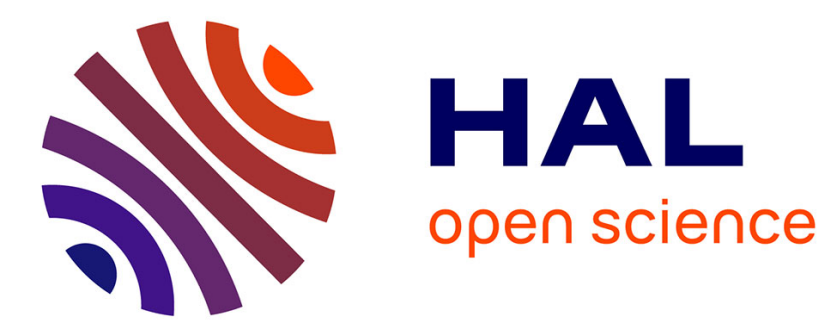

\title{
Grain scale experimental investigation of localised deformation in sand: a discrete particle tracking approach
}

Edward Andò, Stephen A. Hall, Gioacchino Cinno Viggiani, Jacques M Desrues, Pierre Bésuelle

\section{To cite this version:}

Edward Andò, Stephen A. Hall, Gioacchino Cinno Viggiani, Jacques M Desrues, Pierre Bésuelle. Grain scale experimental investigation of localised deformation in sand: a discrete particle tracking approach. Acta Geotechnica, 2012, 7 (1), pp.1-13. 10.1007/s11440-011-0151-6 . hal-01571111

\section{HAL Id: hal-01571111 \\ https://hal.science/hal-01571111}

Submitted on 1 Aug 2017

HAL is a multi-disciplinary open access archive for the deposit and dissemination of scientific research documents, whether they are published or not. The documents may come from teaching and research institutions in France or abroad, or from public or private research centers.
L'archive ouverte pluridisciplinaire HAL, est destinée au dépôt et à la diffusion de documents scientifiques de niveau recherche, publiés ou non, émanant des établissements d'enseignement et de recherche français ou étrangers, des laboratoires publics ou privés. 


\title{
Grain scale experimental investigation of localised deformation in sand: a discrete particle tracking approach
}

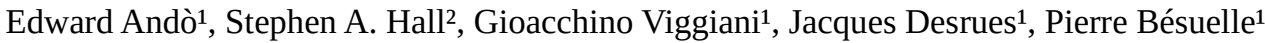 \\ ${ }^{1}$ Laboratoire 3SR (UJF, G-INP, CNRS) ${ }^{2}$ Division of Solid Mechanics, Lund University, Lund, Sweden and \\ European Spallation Source AB, Lund, Sweden
}

Particle Tracking; Granular Media; Shear Bands; in-situ x-ray micro tomography; Experimental Discrete Geomechanics

Recent developments in the application of $x$-ray micro-tomography in laboratory geomechanics have allowed all the individual grains of sand in a test sample to be seen and identified uniquely in 3D. Combining such imaging capabilities with experiments carried out "in-situ" within an imaging set-up has lead to the possibility of directly observing the mechanisms of deformation as they happen. The challenge has thus become extracting pertinent, quantified information from these rich time-lapse 3D images to elucidate the mechanics at play. This paper presents a new approach (ID-Track) for the quantification of individual grain kinematics (displacements and rotations) of large quantities of sand grains (tens of thousands) in a test sample undergoing loading. With ID-Track, grains are tracked between images based on some geometrical feature(s) that allow their unique identification and matching between images. This differs from Digital Image Correlation (DIC), which makes measurements by recognising patterns between images. Since ID-Track does not use the image of a grain for tracking, it is significantly faster than DIC. The technique is detailed in the paper, and is shown to be fast and simple, giving good measurements of displacements, but suffering in the measurement of rotations when compared to Discrete DIC. Subsequently, results are presented from successful applications of ID-track to triaxial tests on two quite different sands: the angular Hostun sand and the rounded Caicos Ooids. This reveals details on the performance of the technique for different grain shapes and insight into the differences in the grain-scale mechanisms occurring in these two sands as they exhibit strain localisation under triaxial loading.

\section{Introduction}

Recent trends in geomechanics research reveal an increasing interest in the small scale mechanisms responsible for the large scale behaviour of granular materials (e.g., Baudet \& Bolton 2010, Jiang et al. 2010, Tejchman \& Górski 2010). Experimental investigation of small scale deformations in geomaterials means transcending the traditional macroscopic measurements and performing measurements throughout a sample volume on some smaller and mechanically-pertinent scale. Emergent small scale phenomena, such as shear strain localisation, are observed in sand with characteristic feature lengths of tens of grains (e.g., Roscoe 1970, Vardoulakis \& Sulem 1995) suggesting that the relevant small scale for the study of these mechanisms is that of single sand grains.

$\mathrm{X}$-ray micro-tomography allows 3D imaging at sufficiently high spatial resolution to see all the individual grains in a sample of sand of appropriate dimensions - see Hall et al. (2010). Furthermore, tomographic scans can be performed in-situ (i.e., at several stages during loading of a sample). Once such 4D (3D + time) images are acquired, the challenge is to make pertinent, quantitative measurements from these images. The study of each grain of sand from a single x-ray tomography image (3D analysis) requires grains to be individuated in a volume, after which their features can be measured numerically (e.g., Wang et al. 2004, Wang et al. 2007, Alshibli \& Alramahi 2006 and Matsushima et al. 2007). Our previous work on the kinematics of sand samples (4D analysis) has used Digital Image Correlation (DIC) to make quantitative measurements such as strain fields with high levels of accuracy. Such approaches are powerful, but treat the medium as a continuum. More recently, discrete analysis of grains kinematics has been performed using Discrete DIC, which for example highlighted the important role of grain rotations in strain localisation (Hall et al. 2010). This approach recognises individual grains based on their "image" (i.e., the greyscale information of the several thousands of voxels which each grain is composed of) which is computationally expensive. An alternative approach (originally proposed by Hall et al. 2008, but not developed), which can be considered closer to Particle Tracking Velocimetry, is to follow the individual grains based on some measurable features, such as their 3D volume (due to the availability of highly optimised image processing packages, tomographic images can be processed in a matter of hours to separate and measure grains). This paper develops this technique, called "ID-Track". It should be noted that a similar approach has been independently explored by Smit (2010).

Discrete analyses of kinematics in sand (such as Discrete DIC and the ID-Track technique proposed in this paper) bring together the 3D analysis of individual sand grains, and the work on kinematics. Discrete grain kinematics are interesting not only as a measurement tool for the small scale of a sand, but also as the first step to compare experimental results with numerical simulations using Discrete Elements and rigid-grain dynamics 
(e.g., Wang 2009).

ID-Track has been applied to the analysis of grain kinematics in several experiments on different granular materials. In this paper, results from two such tests (triaxial compression) are presented and analysed in order to prove the method. This paper briefly introduces the experimental setup and the materials studied, then details the ID-Track method and illustrates its capabilities.

\section{Experimental Setup}

\subsection{Materials studied}

Two different sands are studied in this work. The first is the Hostun HN31 sand, a successor to the RF and S28 varieties that have been extensively studied in Grenoble (e.g., Desrues and Viggiani 2004) - and to which HN31 sand is a priori mechanically identical. HN31 is an angular quartz sand with a $\mathrm{D}_{50}$ of $338 \mu \mathrm{m}$ (as given by the manufacturer). The other material is a sand made of ooids from Caicos, a natural material made up of round calcite grains, whose $D_{50}$ is $420 \mu \mathrm{m}$. Figure 1 shows two sections from x-ray images of both sands, where the difference in angularity is qualitatively apparent.

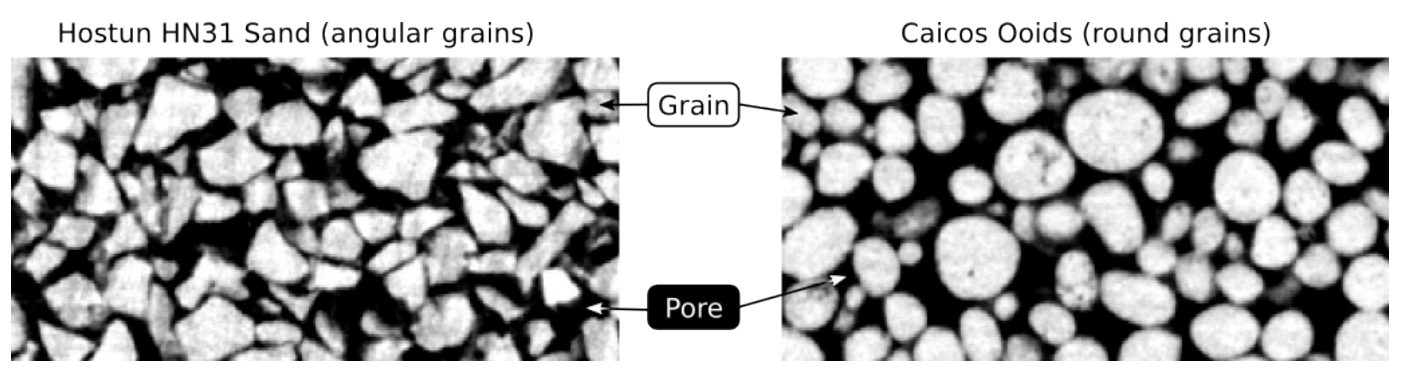

Figure 1 - 2D slices extracted from 3D x-ray tomography volumes of Caicos Ooid sand and Hostun HN31 sand

\subsection{Experimental Technique}

The experiments analysed in this paper are triaxial compression tests on dry material, performed at $100 \mathrm{kPa}$ confining pressure. In each case the samples are prepared by pluviation into a latex membrane stretched in a mould, and measure $11 \mathrm{~mm}$ in diameter by $22 \mathrm{~mm}$ height. The resulting compacity is initially dense. The tests are performed in-situ in the $\mathrm{x}$-ray scanner at Laboratoire 3SR, which allows for scanning of the sample through a full $360^{\circ}$ rotation without displacing the system or unloading (this can be done with or without pausing the loading, in this case loading is interrupted, but the sample is not unloaded). For details of the triaxial system, used previously with synchrotron x-ray tomography, see Hall et al. (2010). Scans at Laboratoire 3SR differ from those performed with synchrotron $\mathrm{x}$-rays principally due to the use of a cone-beam, which implies some differences in the reconstruction technique - the resulting quality and spatial resolution of the 3D images is nonetheless comparable. The voxel size of the 3D tomographic images is in the order of $15 \times 15 \times 15 \mu \mathrm{m}^{3}$, which allows individual grains to be clearly identified (since the $D_{50}$ of the materials studied are between 200 and 400 $\mu \mathrm{m})$ with 3000-15000 voxels per grain in 3D.

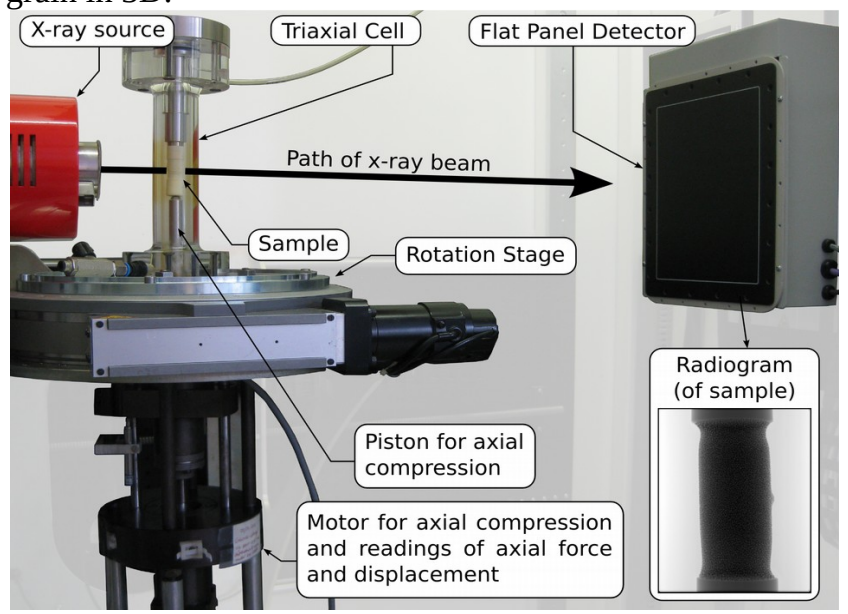

Figure 2 -Laboratoire 3SR Triaxial Setup - in-situ in the x-ray scanner 


\section{ID-Track Technique}

This section outlines the newly developed particle tracking technique "ID-Track" and details the required steps of data processing of $\mathrm{x}$-ray images.

\subsection{Requirements}

The objective of the ID-Track technique is to track individual particles' kinematics (in 2D or 3D). The fundamental requirement for the technique is that the grains are individually identifiable in any two different configurations (a reference and a deformed configuration) and must also be sufficiently different from each other (at the resolution of observation) to allow them to be distinguished from their neighbours by a measurable feature, such as their volume or surface area. The features selected for tracking must be persistent between configurations. Other than these two requirements, two main hypotheses underlie ID-Track: that particles themselves do not deform, and that particles are persistent (i.e., do not break apart or merge). There are no other assumptions on the kinematics of the particles being tracked.

\subsection{Preparation of $x$-ray tomography volumes}

3D x-ray tomography images represent the x-ray absorption field in a scanned volume (Figure 3), and contain no inherent information about individual grains. For measurements of grain characteristics, each grain must be identified and separated from its neighbours in the 3D images. Processing is therefore required to isolate individual grains, as described in the following.

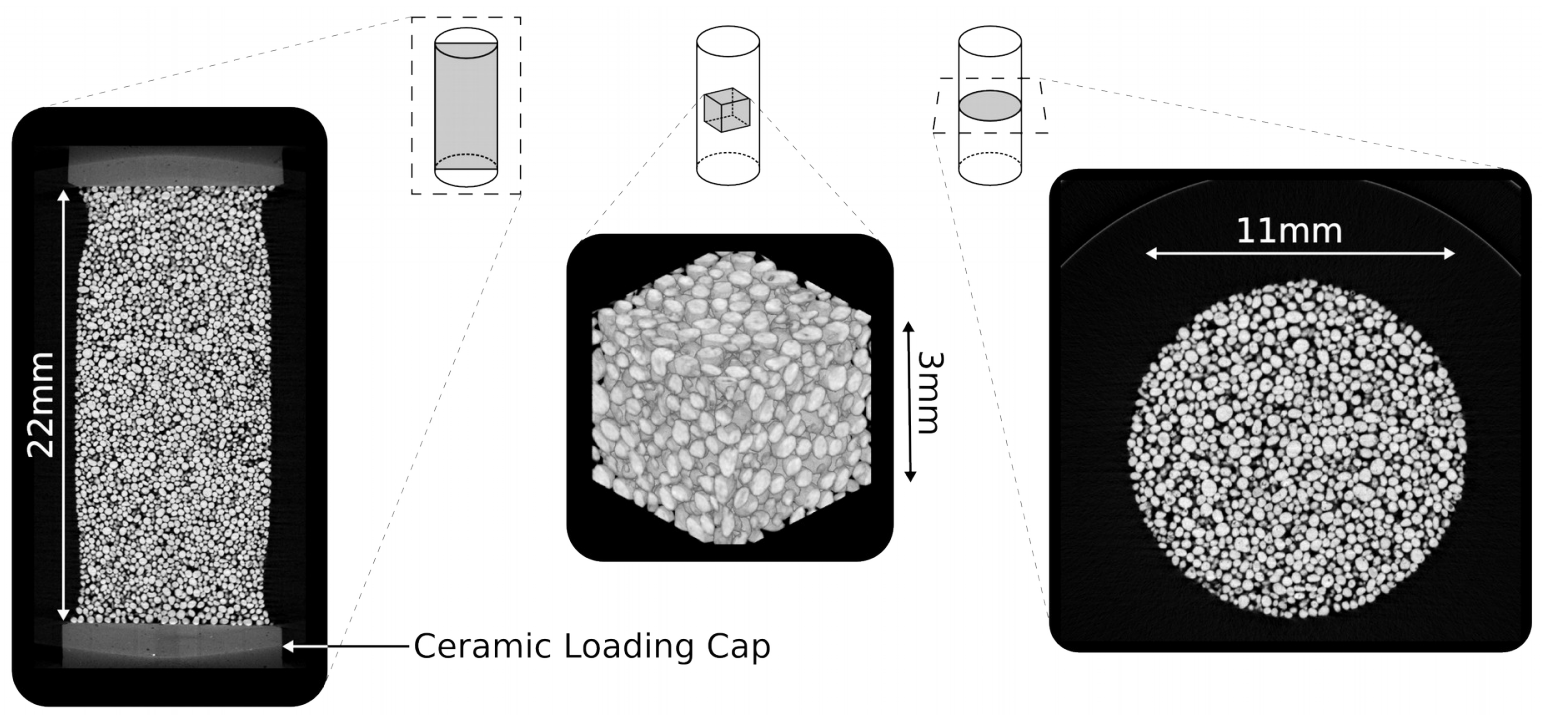

Figure 3 - Two slices and a 3D rendering from an x-ray tomography of a Caicos Ooid sand sample under confining pressure. Lighter colours mean higher x-ray attenuation

In the first step of processing, 3D images are binarised by selecting a threshold value of $\mathrm{x}$-ray attenuation that separates voxels into either grain or not-grain (pore). The threshold is chosen to yield a total number of grain voxels corresponding to the total volume of grains in the specimen as measured physically after the test (by calculation using the known density of the grains and the measured mass of all the grains in the sample). The particles in the resulting binary images are then separated based on their geometry. This process (segmentation) is performed using a high speed 3D Watershed algorithm implemented in Noesis' Visilog 6.910, based on Digabel \& Lanteujoul (1977), which runs in under two hours on a full tomography volume. The separated particles can then be labelled by giving all adjacent voxels (which make up a grain) a unique number, the grain's "ID". Each grain's volume, surface area, centre of mass, and orientation can then be calculated by working on the labelled set of voxels belonging to that grain, as in Figure 4. The whole treatment process is outlined in Figure 5. 

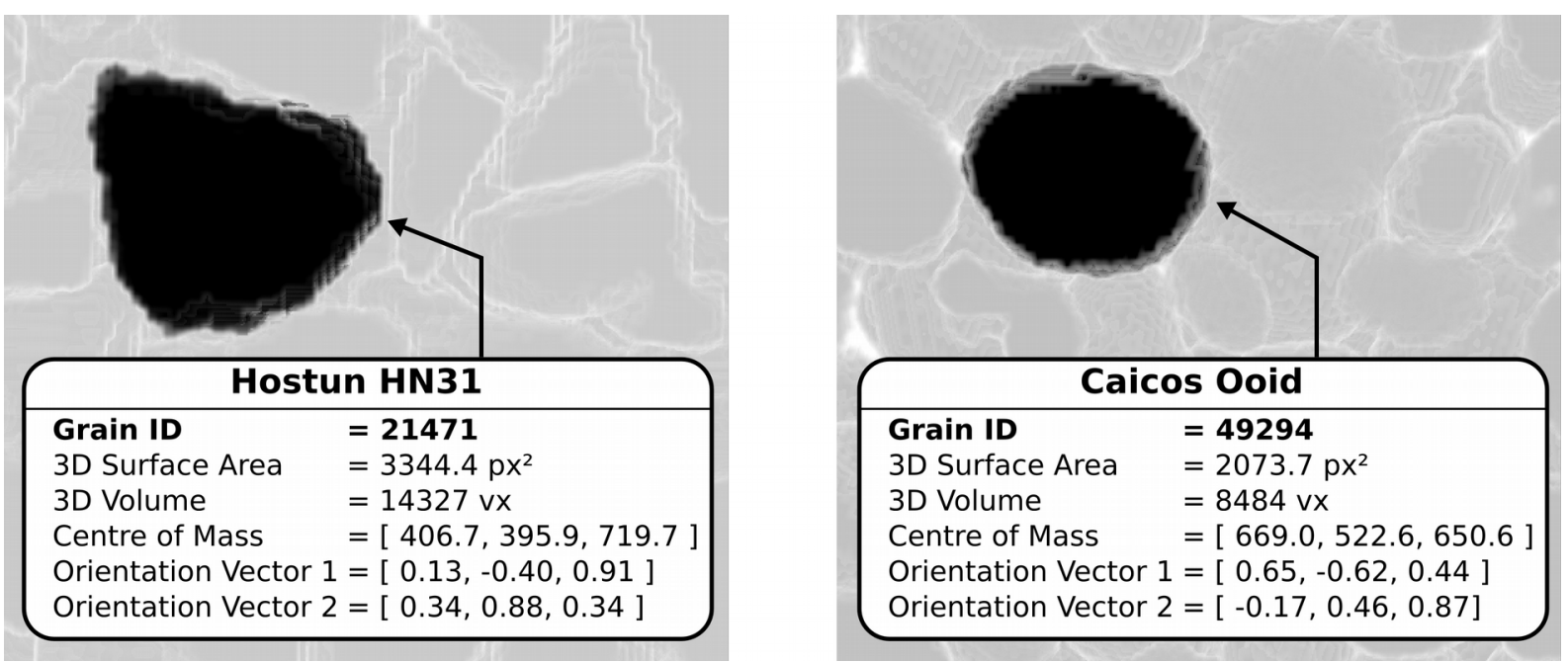

Figure 4 - Rendering in 3D of a single grain of Caicos Ooid sand and Hostun HN31 sand (decimal values truncated in interests of space)

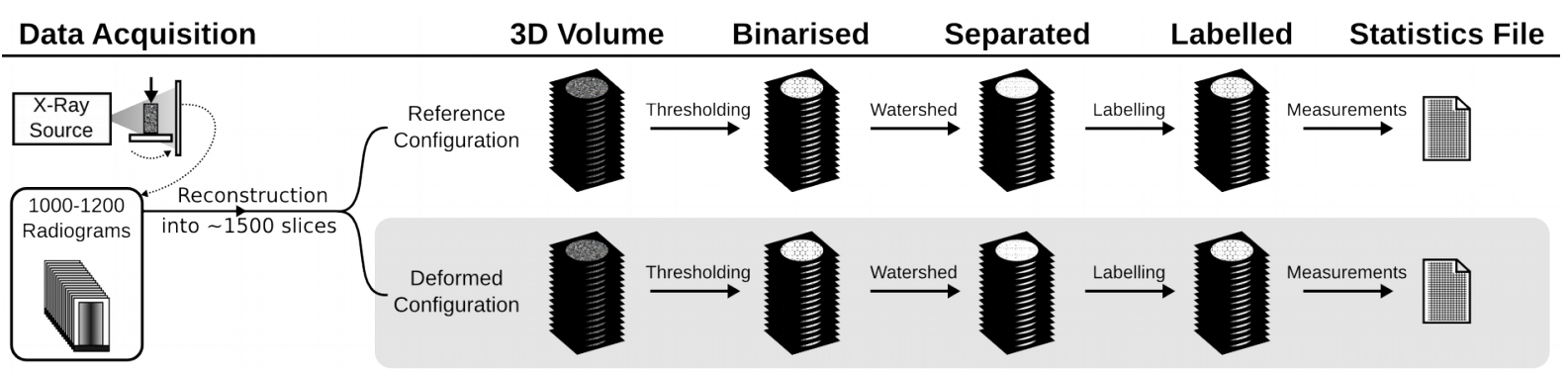

Figure 5 - Data Flow for Labelling Grains

Labels are automatically assigned to grains in each configuration. The same grain in different configurations is therefore unlikely to have the same label. In order to follow grains during a test it is therefore necessary to pair up every grain in the reference configuration to the corresponding grain in the deformed configuration. This is where ID-Track comes in: its first step is the tracking of grains, resulting in the mapping of labels in the reference configuration to the deformed one. With this mapping kinematics can then be determined.

\subsection{ID-Track process}

ID-Track starts by discretising the voxel space in the tomographic image into regular cubes ("neighbourhoods" $50 \times 50 \times 50$ voxels is used in this work). The grains are tracked in two passes: a first rough tracking in order to get mean "neighbourhood" displacements, and a second pass whereby grains are tracked taking into account these neighbourhood displacements. Figure 6 describes the process graphically.

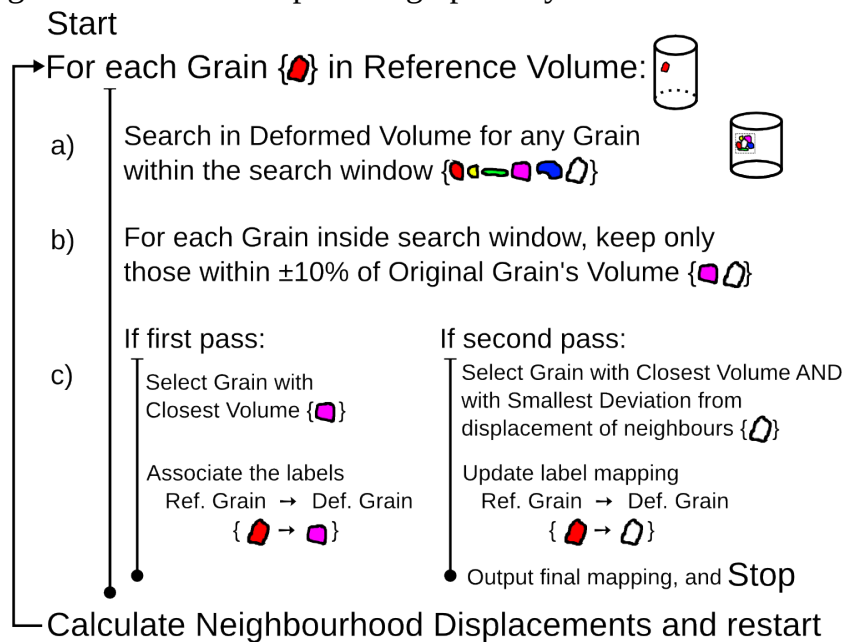

Figure 6 - ID-Track Flow Diagram 
For each grain in the reference volume, potentially corresponding grains are selected in the deformed volume if their centre of mass falls inside the search window (Figure 6a - defined as a certain number of pixels \pm in $\mathrm{X}, \mathrm{Y}$, $\mathrm{Z}$ ). Grains are ignored if the feature used to recognise them (e.g., their volume) is outside \pm a certain tolerance (e.g., 10\%) of the reference grain's selected feature (Figure 6b). In the first pass, the grain in the deformed configuration is simply chosen as the one with the most similar selected feature. In the second pass (Figure 6c) the grain in the deformed configuration is chosen as before but weighting the similarity by how far the displacement this mapping gives is from the median displacement of the neighbourhood. This corresponds to the assumption that grains will tend to displace like their neighbours (note that this may cause problems when a neighbourhood overlaps a developed shear band). For this work a single feature (3D Volume in voxels) has been selected to identify the grains, due to its sufficiently wide distribution (see Figure 7); extension of the technique to include more than one feature to recognise the grains from their ID is possible.
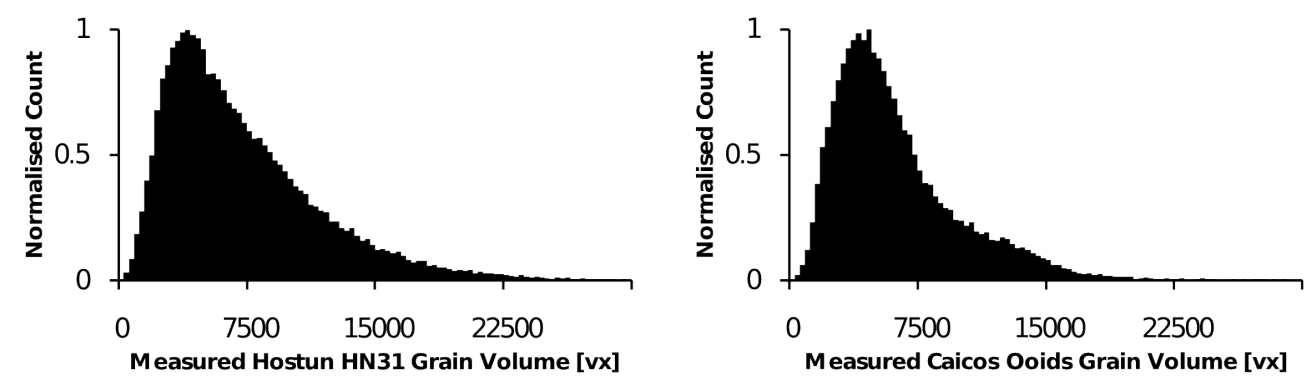

Figure 7 - Distribution of particle volumes (in voxels) for a sample of Caicos Ooids (left) and Hostun HN31 sand (right).

The principal merit of ID-Track is its simplicity, born of its intuitive way of considering particles. Cartoonish as it is, Figure 6 in fact fully details the relevant steps of the algorithm. Nevertheless, the actual implementation of ID-Track involves many technical details such as error checking for input and output data, which are outside the scope of this paper.

The tracking (as described above) does not necessarily ensure a one-to-one mapping of grains between configurations. The case may arise that two grains in the reference configuration are assigned to the same grain in the deformed one. Such conflicts are resolved at the end of the tracking process - the best match is chosen and the grain loosing the conflict is re-tracked.

\subsection{Determination of Grain Kinematics}

Once a mapping exists between grains from one configuration to another, the kinematics of each grain can be obtained. 3D displacements of grains are simply measured from the difference in 3D position of their centres of mass. Rotations of grains require them also to be fully oriented in space; in 3D this requires two orientation vectors to be defined for each grain. Orientation vectors are defined by calculating the moment of inertia tensor for the voxels making up the grain, whose eigenvectors are the principal axes of inertia. Visilog provides two orthogonal and unit-length vectors $(\vec{L}$ and $\vec{W}$ ) calculated from the maximum and minimum eigenvectors of the moment of inertia tensor. These vectors give a grain's orientation, and an orthonormal base is obtained by calculating a third vector $\vec{D}$, which will be the intermediate axis. These are all determined for both the reference (ref) and the deformed (def) configurations. With this orthonormal base in the reference and deformed configurations, the matrix $\boldsymbol{R}$, describing the rotation of the grain from one configuration to the next is given by:

$$
\boldsymbol{R}=\vec{L}_{\text {def }} \otimes \vec{L}_{\text {ref }}+\vec{W}_{\text {def }} \otimes \vec{W}{ }_{\text {ref }}+\vec{D}_{\text {def }} \otimes \vec{D}_{\text {ref }}
$$

In order to simplify the interpretation of these rotations, the rotation matrix $\boldsymbol{R}$ is represented as a single axis of rotation around which the grain rotates. The null space of the rotation matrix (Vector $\mathrm{x}$ for which $\boldsymbol{R x}=0$ ) gives the axis of rotation. In order to measure the scalar value of rotation around the axis of rotation, a vector normal to the axis of rotation is rotated by $\boldsymbol{R}$. Since the rotation is measured by a dot product and the axis of rotation is not fixed in space, the largest rotation that can be measured is $180^{\circ}$. Furthermore, since no sense is associated to the orientation vectors (which can be represented by any of two equal and opposite vectors), a $180^{\circ}$ flip sometimes occurs. This is corrected, for flips of the principal axes, by folding the rotations around $90^{\circ}$ : for example a real $3^{\circ}$ rotation which experiences a flip will be measured as $177^{\circ}$, and is brought back to $3^{\circ}$ by folding. All the rotations r undergo the following operation:

$$
\mathbf{r}_{\text {folded }}=|| \mathbf{r}-\mathbf{9 0}^{\circ}\left|-\mathbf{9 0}^{\circ}\right|
$$

Note that this implies that only rotations between 0 and $90^{\circ}$ can be measured with this approach. 


\subsection{Measurement and tracking errors}

Sources of error identified in the tracking of grains in the tests analysed are principally related to either limitations in the technique (an incorrect tracking can occur if there are two grains with similar features close to each other) or "upstream" errors in the data treatment process (especially if grains are badly separated in the segmentation of the tomographic volume). The quality of the watershed process used to separate grains works best on spherical grains, so if grain shapes are far away from a sphere, non-physical separations can occur. Grains can either be too separated (oversegmented, see Figure 8a) or not separated enough (undersegmented, see Figure 8b). Since segmentation errors are unlikely to occur predictably between different configurations, these "grains" cannot be tracked, because their selected feature will change too much.
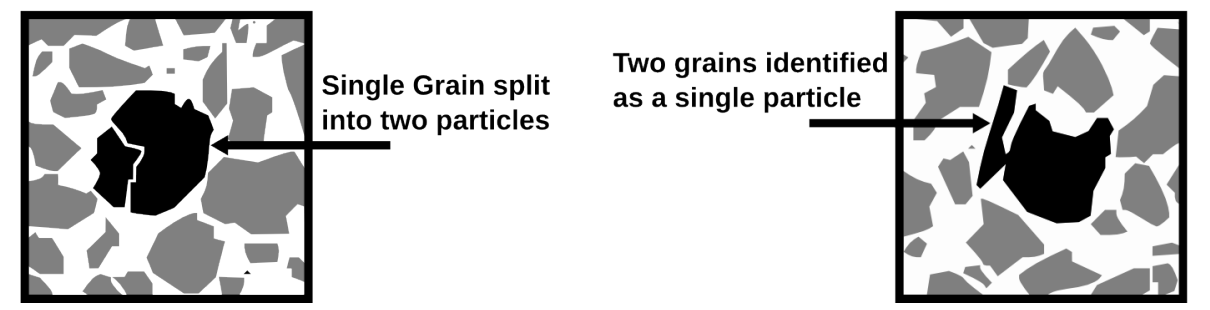

Figure 8 - Binarised images of non-physically segmented grains (Hostun HN31 Sand)

If a grain cannot be tracked (no sufficiently similar grains in its neighbourhood), this situation is detected, meaning that remedial measures could be put in place to join oversegmented grain parts together or to separate an undersegmented agglomeration of particles. A foreseeable challenge is the detection whether a tracking error is due to over or undersegmentation - a possible source of information to distinguish between these situations could be to compare the volume or curvature of the particles to a reference value for the studied material. Table 1 summarises major sources of error identified so far.

\begin{tabular}{|l|l|l|}
\hline \multicolumn{2}{|c|}{ Error Sources } \\
\hline \multicolumn{1}{|c|}{ Source } & Description & \multicolumn{1}{c|}{ Diagnosis } \\
\hline $\begin{array}{l}\text { Imaging System (image } \\
\text { acquisition) }\end{array}$ & $\begin{array}{l}\text { displacement (e.g., } \\
\text { thermally induced) of } \\
\text { imaging system with } \\
\text { respect to sample }\end{array}$ & $\begin{array}{l}\text { Adds a bias to the image - this can be } \\
\text { treated in the kinematic analysis }\end{array}$ \\
\hline $\begin{array}{l}\text { Image Pre-Processing (from } \\
\text { greyscale to binary image) }\end{array}$ & Binarisation & $\begin{array}{l}\text { Causes jagged edges and loss in definition } \\
\text { of boundaries (since only denser partial } \\
\text { volume voxels will be selected) - loss in } \\
\text { precision of grain shape is not major }\end{array}$ \\
\hline $\begin{array}{l}\text { Image Pre-Processing (from binary } \\
\text { image to individually labelled } \\
\text { grains) }\end{array}$ & $\begin{array}{l}\text { Errors in segmentation } \\
\text { (grains either stuck } \\
\text { together or single grain } \\
\text { split apart) }\end{array}$ & $\begin{array}{l}\text { Causes major problems for tracking, see } \\
\text { below - could be resolved by ID-Track, but } \\
\text { requires a lot of } \text { a priori knowledge }\end{array}$ \\
\hline ID-Track & Grains not tracked & $\begin{array}{l}\text { Causes “holes" in data, but as long as the } \\
\text { error rate is low (approximately 5\% in the } \\
\text { data presented herein) this does not impede } \\
\text { interpretation of results }\end{array}$ \\
\hline ID-Track & Grains incorrectly tracked & $\begin{array}{l}\text { Results in incorrect results for a few grains } \\
\text { dispersed over the specimen, which may } \\
\text { complicate (but does not jeopardise) the } \\
\text { analysis of the overall behaviour }\end{array}$ \\
\hline
\end{tabular}

Table 1 - Principal sources of error

\subsection{Metrological Aspects of ID-Track}

As described above, grains are imaged in 3D by x-ray tomography, then binarised and segmented (i.e. separated from each other). In order to be able to give a total value of accuracy, the metrological analysis should include characterisation of the image acquisition system, but since this is unique to each imaging setup, this will not be done here. However, it is worth noting that a varying bias in the centring of the image has been observed with the imaging setup used (see Table 1). These variations are thought to be principally caused by differential 
displacements in the imaging setup, related to temperature variations. These effects can, at least in part, be corrected during the image reconstruction.

The key measurements made by ID-Track are displacements and rotations of particles. The displacements are the difference in a particle's centre-of-mass position between configurations. Rotations are calculated from a difference in orientation of the eigenvectors of the grain moment-of-inertia tensor between configurations. Since centres of mass and orientations are calculated entirely independently (and can thus be improved independently) the metrological aspects of the measurement of displacements and rotations will be presented separately.

\subsubsection{Displacements}

Displacements are calculated from a difference in position between reference and deformed configurations. The position in a given configuration is given by a calculation of the centre of mass of the (binarised) particle. Thus the metrological aspects of the definition of the centres of mass will govern the precision of the displacement measurements. This is discussed further below.

The centre of mass of each grain is calculated as a positional average of the several thousand binary voxels (see Figure 7) that compose the grain. In the literature of digital image measurement, a great deal of attention has been paid to finding analytical results for simple, 2D geometrical shapes known a priori, such as pixelised lines (e.g. O'Gorman 1996), points (Havelock 1989), circles (Nakamura \& Aizawa 1984, Landau 1987). Because of the predictable nature of the pixel patterns of straight lines and the known symmetry of circles, there is a large quantity of a priori information that can be integrated into a measurement. A useful idea proposed by Havelock (1989, 1991) is that of "locales" which describing the regions in pixel- or voxel-space in which a shape can move while its binarised image stays the same. Adding the calculation of the centre of mass to this concept, it may be possible to characterise the sensitivity, precision and accuracy with which a shape can be placed in 3D. This is highly desirable, but again requires "perfect" geometrical knowledge of the shapes under study. These analytical solutions, and the concept of locales are unfortunately not suitable, at least in their current form, for the characterisation of the measurements made on the particles treated in this work for three main reasons: first, the natural grains studied in this work don't have perfect, symmetrical geometrical shapes; second, each grain is unique (there is no accurate and generalised description of grain shape which can be given to all the grains of Hostun sand for example); and last, a variety of different grain shapes is used in this work.

In order to estimate the possible error in the positions of the centre of mass of the grains measured, some best and worst case scenarios can be identified. Consider the binarised image of a 1D line; it can be shown that the position of the centre of mass depends only on the position of the first and last pixels of the line ${ }^{1}$ (the boundary). This means that the measurement of the centre of mass of a line whose image is binarised has both sensitivity and maximum error of 0.5 pixels. Extrapolating to 2D shapes, if a shape is broken down into its component $1 \mathrm{D}$ lines, in the worst case (if all the 1D lines are aligned - i.e. a square), the sensitivity and maximum error in the definition of the centre of mass, in the direction of the lines will remain 0.5 pixels. The best case is that the $1 \mathrm{D}$ lines are laid out in such a way that their ends are distributed equally between 0 and 1 pixels - this would mean that the sensitivity is improved cumulatively, i.e. 0.5 / (number of $1 \mathrm{D}$ lines). For 3D shapes with volume 5500 voxels the worst case would be a cube, aligned with the reference system of the image - in which case again the sensitivity and maximum error would be 0.5 pixels. The best case would be a shape, specially tailored to have an equal distribution of grey pixels when it is imaged in any position. Supposing that is it close to a sphere, the radius would be approximately 11 pixels, and the cross-sectional area would be: 353 pixels ${ }^{2}$, which means 353 separate $1 \mathrm{D}$ lines in any direction. The best case sensitivity would then be $0.5 / 353=0.0014$ pixels. Since in practice the best case sensitivity requires a carefully calibrated shape, a simulation of the displacement of a real sphere (radius 11 pixels) from 0 to 1 pixels with 300 steps is performed. This geometrically defined shape is discretised onto a 3D voxel grid, where voxels are assigned 1 if they are inside the shape and 0 if they are outside. Boundary voxels are integrated with $6^{3}$ points inside each voxel to give a value between 0 and 1 - these boundary voxels are then binarised with a threshold of 0.5 . The worst case error observed in this case is 0.03 pixels (mean error is 0 pixels; mean absolute error is 0.012 pixels). This immediately shows that the maximum error in the position of the centre of mass of a 5500 voxel sphere is far from the best case. Since the centre of mass depends only on the position of voxels on the boundary, one can say that for a sphere this size, the $353 \times 2$

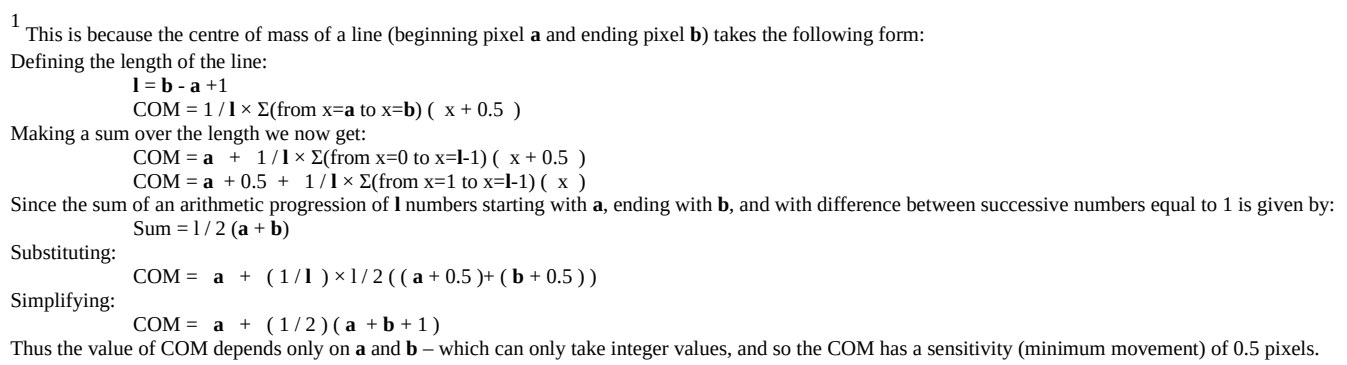


boundary voxels are not numerous enough to ensure that their greyvalues (before binarisation) were welldistributed with values from 0 to 1 . This in turn means that they are not "equiprobable", which explains the difference between this maximum error and that predicted by the best case. It is expected that the larger the surface becomes, the closer the maximum error will be to the best case maximum error (for a given, nonstraight-lined shape). The sphere is chosen for the numerical simulation for its simple mathematical definition and not for its symmetrical properties - the error shown above should hold approximately for non-straight-lined shapes of approximately the same surface area. This means that the measurements of displacements (accumulating twice the error of the measurement of the centre of mass), should have a worst-case error of approximately 0.06 pixels for each component of the 3D measurement of displacements.

Many techniques are possible in order to improve the accuracy of the measurement of the centre of mass, and they fall broadly into two categories: improvements on the binary image of the grain, or "greyscale" improvements. Techniques in the first category work from the binary image of the grain, and could attempt to improve the measurement by either (1) smoothing the jagged surface, either by curve fitting with a 3D technique such as NURBS or using a marching cubes algorithm, or (2) weighting the boundary of the binary grain less than the centre of the grain, to artificially make a smoother transition at the boundary. Otherwise a "greyscale" approach means looking up the positions of the binary voxels that make up the particle in the original reconstructed greyscale volume - and using these to calculate the centre of mass - taking advantage of the less jagged partial volume voxels on the border of the grain.

In theory, the calculation of a centre of mass from a binary image may be potentially as precise as Discrete DIC, in that both methods essentially rely on the boundaries of the grains. DIC has the potential to be more precise than ID-Track because internal contrast inside the grains might give extra information (which is essentially flattened in ID-Track's binarisation process). However, as reported by Pannier et al. (2010), the contrast inside the grains cannot be relied upon in the x-ray tomography images acquired during these tests: the variations seen inside the grains are largely dominated by noise - they report a much larger total experimental error of 0.1 voxels in translation. In any case, the maximum error reported in this work is deemed satisfactory for the analysis of the phenomena under observation.

\subsubsection{Rotations}

As developed above, rotations are calculated from orientations in a reference and a deformed configuration.

The definition of accuracy is again not straightforward; the moment of inertia tensor is determined on irregular particles, and a few voxels included on one side of a grain may be enough to cause a step change in the orientation of the principal axis of a grain (especially in close-to-spherical ones). The measurement thus relies on a certain degree of anisotropy in the shape of a grain for its success - a perfectly spherical grain's orientation cannot be defined.
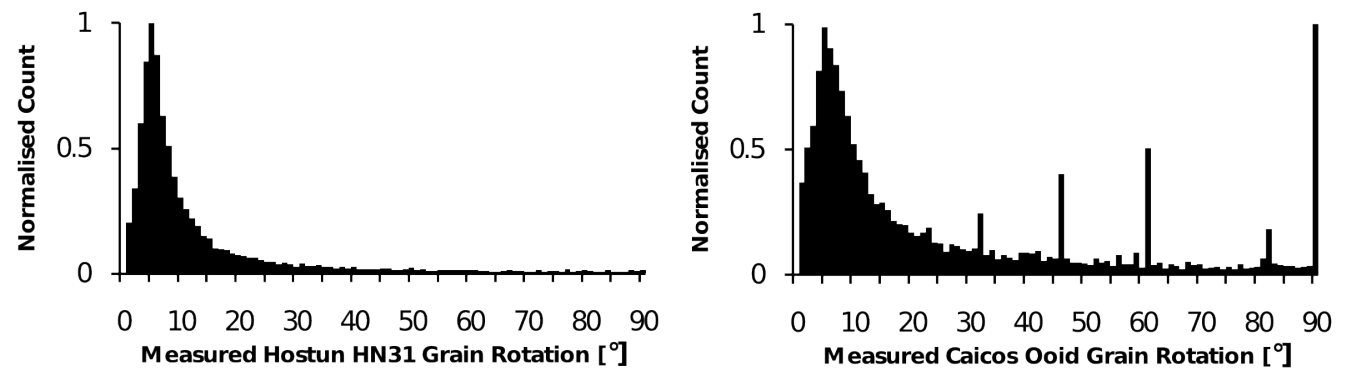

Figure 9 - Distribution of measured grain rotations for Hostun and Caicos sands for an imposed rigid body rotation of the whole specimen of $4.7^{\circ}$

To quantify the accuracy of the measurement of grain rotations, the simple experiment in the style of Pannier et al. (2010), has been performed by applying a rigid body rotation of $4.7^{\circ}$ of the whole specimen between reference and deformed configurations. Figure 9 shows the distribution of grain rotations measured in this experiment, in which different sands were tested; results are shown for the two materials studied. As seen above, rotation of each grain is calculated from the change in orientation of the grain between configurations. Figure 9 indicates that the measurement of rotations is relatively accurate since the distribution is centred on $4.7^{\circ}$ for both sands, however not as precise: the Standard Deviation of the measurements is large $\left(16.4^{\circ}\right.$ for Hostun and $28.3^{\circ}$ for the Ooids). It is worth noting that although rotations can be measured for both sands, the rounder Caicos Ooid sand has a higher error rate, and errors are noticeable at rotations multiple of $15^{\circ}$, which indicate symmetry of the particle. Although this measurement technique is considerably less precise than Discrete DIC (Pannier et 
al. 2010 report a $0.1^{\circ}$ precision for rotations in Hostun sand), compared to the rotations measured it yields results of sufficient quality for the purposes of this investigation into the kinematics of shear bands.

\section{Results}

Results from the application of ID-track on five strain increments for the triaxial tests on the two different sands are shown in Figure 10. Note that for the test on Hostun sand the tracking has only been performed between highlighted strain increments for better comparison with the test on Caicos Ooids, even though many more strain increments exist. The results presented are vertical slices through the labelled volume of grains in the reference state; the slices have been oriented in order to contain both the axis of the sample and the normal to the shear band that eventually forms. Each grain which has been tracked for a given increment is coloured, in the results shown, either according to its displacement (the norm of the displacement vector) or according to its value of rotation (the axis of rotation is not shown). Grains that are not tracked are left blank. Insofar as displacement is concerned, readers are reminded that the sample is compressed from the bottom upwards, so that displacement in the axial direction is imposed as 0 at the top of the sample. 

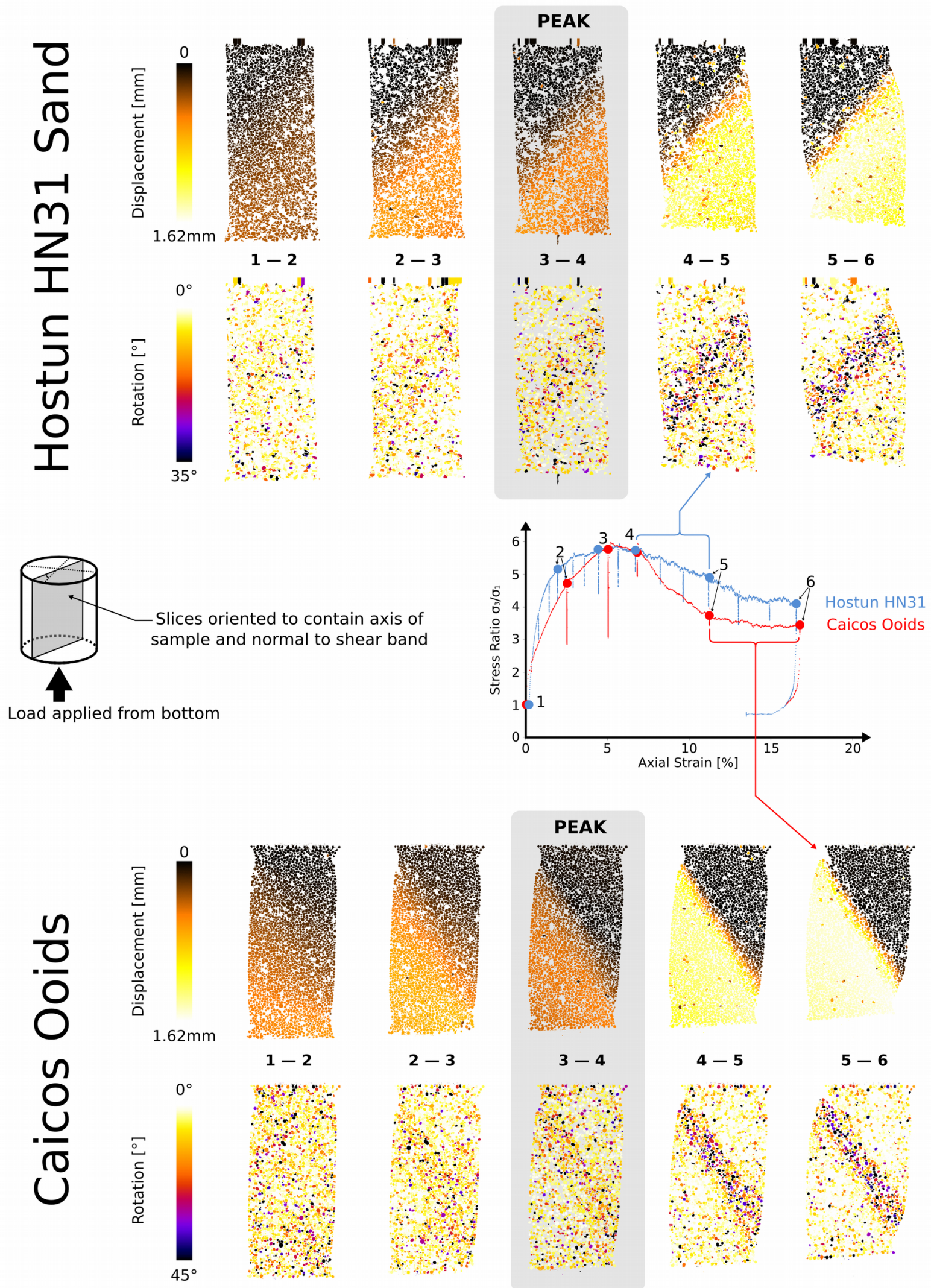

Figure 10 - Results of ID-Track on five strain increments for TXC on Hostun HN31 Sand and Caicos Ooids. Results are shown as slices oriented in order to contain both the axis of the sample and the normal to the shear band that eventually forms; tracked grains are coloured by the norm of their displacement vector or value of rotation (axis of rotation not shown) 
The results presented in Figure 10 show displacements and rotations for all the tracked grains in a slice. It is immediately visible (especially in the displacements) that the overall kinematics of the sample are characterised by smooth fields - as previously shown with continuum DIC (Hall et al. 2010). At the end of both tests, a highly localised pattern of deformation in the form of a single shear band occurs. Although other, more complex patterns of localisation are observed when shearing dense sand in triaxial compression (e.g., Desrues et al. 1996), these are unlikely to occur in the very small specimens (with respect to grain size) tested in this work. In any case, a single shear band is the localisation mode expected for a dense sand sheared with standard conditions (sample with slenderness ratio of 2 and non-lubricated ends) - see for example Desrues et al. (1996).

The test on Hostun sand shows that the tracking can be performed successfully on the images acquired in this test. The displacement field in the first increment of loading Figure 10 (top) appears to be smooth, although there are some grains which are not tracked (6 to 8\% per increment - see Figure 11 left), which correspond to blank grains - i.e. holes in the image. Furthermore, there are occasional grains which are incorrectly tracked: grains with displacements very different to their neighbours can sometimes be seen. The overall behaviour is however clear: over the first strain increment there is an almost smooth incremental displacement, from 0 at the top of the sample (imposed) to $0.4 \mathrm{~mm}$ at the bottom (also imposed). A slight lack of verticality in the sample at the beginning of the test (sample axis tilted by $1.4^{\circ}$ in the slices shown) manifests itself as an "inclined" displacement field relative to the direction of loading. In continuum mechanics this would correspond to a strain field which is not wholly uniform, yet without strong gradients. In the following increment (2-3), before the peak in the overall sample response, the gradient of the displacement field is oriented perpendicular to the direction of the band that eventually forms. These discrete observations support the theoretical idealisation of a shear band as a region of localised deformation bounded by weak discontinuities, i.e., discontinuities in the gradient of displacement. The displacements progressively concentrate into a band 7 to 8 grains thick by the last increment (5-6, see Figure 12, top left for a zoom into the displacement field). The band appears to be oriented at about $47^{\circ}$ from the horizontal.

Up until the peak, rotations appear to be disorganised in space. After the peak, when the band is fully developed, the rotations are clearly concentrated in space. In the increment that lies over the peak (3-4) some concentration of rotations can be discerned. Initial indications of this concentration might exist in increment 2-3. The rotations are concentrated in the same band as indicated by displacement, although the width of concentration appears to be larger (10 to 12 grains thick, see Figure 12, bottom left). ID-Track also provides the axis of rotation for each grain, which is not necessarily orthogonal to the slices shown. It is of interest to look at the component of rotation occurring in the plane of the slices, i.e., the component of rotation around an axis orthogonal to the plane of the slices (which might be termed rolling in the "steepest direction", if the shear band is imagined as a plane on which grains are rolling). It appears that grains inside the band do not show any more evident concentration of rotation when measured with this component - from which we can conclude that rotations clearly concentrate in the band, but apparently not in the "steepest direction".

For the test on Caicos Ooids presented in Figure 10 (bottom) it can be seen that grains are tracked more successfully, with only 1 to 3\% lost grains (see Figure 11 right). This is due to a higher quality of segmentation, since the watershed algorithm works better on the more spherical grains of this material. In terms of displacement, a similar phenomenon to the Hostun sand can be seen to occur - the major differences are that the band appears slightly more concentrated (6 to 7 grain diameters, see Figure 12, top right), and steeper relative to the horizontal $\left(54^{\circ}\right)$. Note that as with the Hostun sample, the displacement field is slightly inclined from the very beginning of the test.

As with Hostun, rotations are disorganised in space and therefore not localised in the first increment. Rotations start to concentrate after the peak, again with hints visible over the peak, and possibly already in the preceding increment (2-3). The band as measured by rotation is thicker than that measured by displacement, but considerably less so than with Hostun sand (7 to 8 grain diameters, Figure 12, bottom right). It should be noted, that although the sphericity of the grains results in a higher quality segmentation, the grains are not so spherical that their orientation cannot be well defined by their moment of inertia tensor. As with Hostun, the rotations localise, but the directions of rotation apparently do not polatise. 


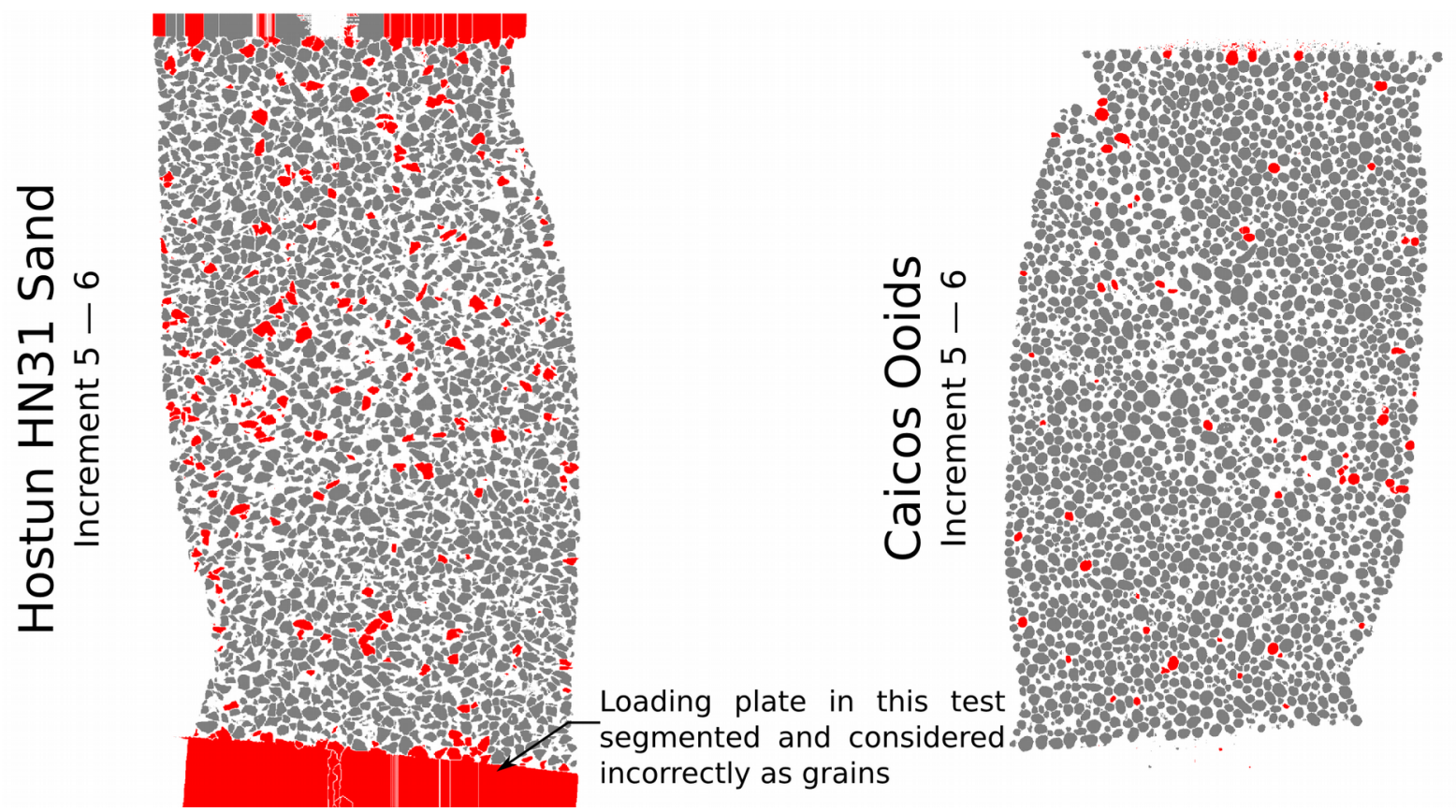

Figure 11 - Untracked grains (in red) for the last strain increments shown in Figure 10

One tentative explanation for the difference between the relative width of the shear band as measured by displacement or rotation for these two sands, is based on the grain shape: the shear band (which is inherently a 2D phenomenon at the sample scale) causes grains to move differentially while it is trying to develop in a 3D material. The development of the band causes a region with a thickness of about 7 grains to displace differentially (the bottom "block" of grains moves almost as a rigid body). However, in order to displace differentially, the grains in the shear band must also rotate around each other. In a rounded material these rotations are not strongly transmitted into the rest of the sample due to the lower level of interlocking between grains, whereas in the angular material, a "cog” effect may occur, explaining why the band measured in rotations is so much thicker for Hostun sand. The greater number of grains involved in the rotations may also explain the higher residual stress of the angular sample, since sliding in the band (necessarily causing rotation in 3D) is "resisted" more strongly. 


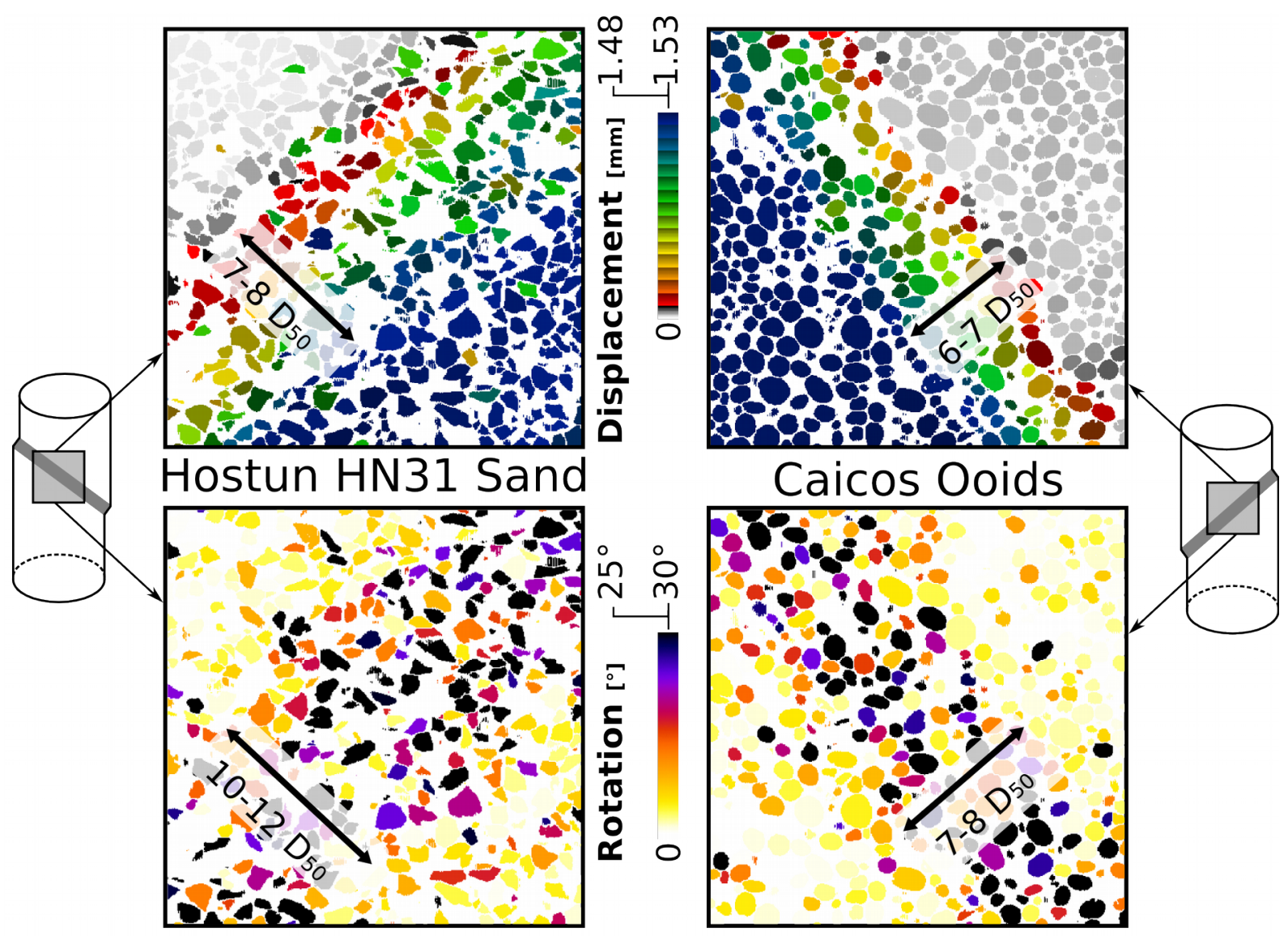

Figure 12 - Zoom into shear band for the two tests, at the last strain increment 5-6 in Figure 10

From a computational point of view, it should be reported that, given the calculated grain IDs and their positions and orientation vectors in space for a reference and a deformed configuration, tracking takes only a few minutes on a powerful laptop. This is a major advantage relative to the reported tens of hours for an increment of Discrete DIC - the significant time saving means that additional computational cycles could be reasonably used to improve the tracking and still keep the calculations times relatively short.

\section{Conclusions}

Recent developments in x-ray micro-tomography have lead to the possibility of non-destructive 3D imaging of materials at spatial resolutions that were simply unimaginable a couple of decades ago. In the current context, this advance permits all the individual grains of sand in a test sample to be seen and identified uniquely in 3D. Combining such imaging capabilities with experiments carried "in-situ" within an imaging set-up has lead to the possibility of observing directly the mechanisms of deformation as they happen. The challenge has thus become extracting pertinent, quantified information from these rich time-lapse 3D images to elucidate the mechanics at play. This paper has presented a new approach for the quantification of individual grain kinematics (displacements and rotations) of large quantities of sand grains (ten of thousands) in a test sample undergoing loading (in this case triaxial loading).

The key contribution of this paper is a new 3D+Time grain-kinematics measurement technique, named "IDTrack" that provides a detailed insight into the grain-scale mechanics of deformation (here shear banding) in granular media with a low computational effort and acceptably low error rate. Grains are tracked between images at different stages of deformation based on some geometrical feature(s) that allow their unique identification and matching between images. 3D displacements of grains are then obtained simply from the difference in 3D position of the centres-of-mass of each tracked grain. Grain rotations are determined from the change in orientation vectors of the grains, defined from the eigenvectors of the moment of inertia tensor for the voxels making up the grain.

The principal merit of ID-Track is its simplicity, born of its intuitive way of considering particles. The key requirement of the method is that the grains are individually identifiable and distinguishable from other grains in any two different configurations (e.g., a reference and a deformed configuration). In addition, it is assumed that 
particles are persistent (i.e., do not break apart or merge) and that they do not deform. The technique is thus ideally adapted for the analysis of low confining pressure triaxial tests on sand (it would not, however, in its current form allow analysis of experiments in which grain breakage, for example, occurs).

Errors in the approach have been identified as arising from (i) incorrect tracking, which can occur if there are two grains with similar features close to each other; (ii) "upstream" errors in the image analysis prior to ID-track, in particular poor segmentation of the grains giving different results for each input image. However, the method is potentially as precise as Discrete DIC, for example. Some quantification of the expected uncertainties in the measured kinematics have been provided. For displacements the uncertainties are in the order of 0.02 pixels. The measurement of rotations has been deemed to be of sufficient quality for the purposes of the current investigation in that they are relatively accurate, although not as precise. It is also noted that the rotation results depend significantly on the form of the grains: the standard deviation of the measurements is $16.4^{\circ}$ for angular Hostun and $28.3^{\circ}$ for the rounded ooids (note that the orientation, and thus rotation, of a perfectly spherical grain cannot be defined).

Results have been presented from successful applications of ID-track to triaxial tests on two quite different sands: the angular Hostun sand and the rounded Caicos Ooids. On the technique side, a key finding is that, in both cases, a single feature of the grains (3D Volume in voxels) is sufficient to individually identify and distinguish the individual grains from one another between 3D images acquired at different loading steps. However, it was also found that the grain-tracking works better for the more rounded Caicos sand, which arises from the better performance of the images segmentation algorithm to separate more rounded grains (the watershed algorithm used works best for spherical grains).

In terms of the mechanical analysis, it has been observed that, for both sands the displacements progressively concentrate into localised bands (with widths of 7-8 grains for Hostun and 6-7 grains for Caicos). The rotations appear to be disorganised in space until the peak stress after which the localisation band becomes fully developed and the rotations are clearly concentrated in space in the same band as indicated by displacements, although the width of concentration appears to be larger (10-12 grains thick for Hostun and 7-8 grains wide for Caicos). This difference in the relative band widths from displacements and rotations for the two sands suggests a difference in the grain interactions due to different grain shapes: for the rounded Caicos grains there is a lower level of grain interlocking, whereas the angular Hostun grains potentially exhibit a "cog" effect may occur, which transmits the rotations further from the localised zone. No polarisation of the rotations with the band orientation is observed in either case.

Work is ongoing to improve the technique - both to reduce incorrectly tracked grains and to resolve the issue of incorrect grain segmentation. As compared to Discrete DIC (previously proposed in Hall et al. 2010) ID-Track is simpler to implement, significantly faster and potentially as precise in the measurement of displacements. Discrete DIC however looses fewer grains and measures rotations more precisely, over a larger range and does so more reliably. Discrete DIC furthermore depends less crucially on a correct segmentation. ID-track might be improved by using more grain features in combination for the tracking or by utilising different means to more accurately quantify the grain centres-of-mass and orientation (e.g., using the grey-scale information as opposed to just binary images). However no technique is perfect, so a combination of ID-Track and DIC approaches is envisaged, as proposed in Hall et al. (2008), to benefit, for example, from the computational simplicity of IDtrack and from the more accurate and precise measurement of rotations from DIC. Finally it is noted that similar data to those presented herein also exist for the same materials at different confining pressures and also for other granular materials (Ottawa sand and glass ballotini); the results of this experimental campaign will be the object of forthcoming publications.

\section{References}

Alshibli, K. A., Alramahi, B. A. (2006). Microscopic Evaluation of Strain Distribution in Granular Materials during Shear. J. Geotech. Geoenviron. Eng., 132(1), 80-91

Baudet, B., \& Bolton, M. (Eds) (2010). Soil mechanics at the grain scale: issues 1 and 2 (Themed Issue). Géotechnique, 60, 5-6.

Desrues, J., Chambon, R., Mokni, M., Mazerolle, F. (1996). Void ratio evolution inside shear bands in triaxial sand specimens studied by computed tomography. Géotechnique, 46, 3, 529-549

Desrues, J., Viggiani, G. (2004). Strain localization in sand: an overview of the experimental results obtained in Grenoble using stereophotogrammetry, International Journal For Numerical And Analytical Methods In Geomechanics, 28, 4, 279-322

Digabel, H., \& Lanteujoul, C. (1977). Iterative algorithms. Proc. 2nd Europ. Symp. on Quantitative Analysis of Microstructures in Material Science, Biology and Medicine. Caen, France.

Hall, S.A., Lenoir, N., Pannier, Y., Desrues, J., Bornert, M., Viggiani, G., Bésuelle, P., Di Michiel, M., and Otani, 
J. (2008), Characterisation of localised deformation in granular geomaterials using x-ray (micro)tomography and 3D-volumetric digital image correlation [abstract], Photomechanics 2008, 7th - 9th July 2008, Loughborough, UK.

Hall, S.A., Bornert, M., Desrues, J., Pannier, Y., Lenoir, N., Viggiani, G., Bésuelle, P. (2010). Discrete and continuum experimental study of localised deformation in Hostun sand under triaxial compression using X-ray $\mathrm{CCT}$ and 3D digital image correlation. Géotechnique, 60, 315-322.

Havelock, D. I. (1989). Geometric Precision in Noise-Free Digital Images. IEEE Transactions on Pattern Analysis and Machine Intelligence, 11, 10, 1065-1075

Havelock, D. I. (1991). The Topology of Locales and Its Effects on Position Uncertainty. IEEE Transactions on Pattern Analysis and Machine Intelligence, 13, 4, 380-386

Jiang, M., Liu, F., \& Bolton, M. (Eds.) (2010). Geomechanics and Geotechnics: From Micro to Macro. ISShanghai 2010. Shanghai: CRC Press.

Landau, U. M. (1986). Estimation of a circular arc center and its radius. Computer Vision, Graphics, and Image Processing, 38, 317-326

Matsushima, T., Katagiri, J., Uesugi, K., Tsuchiyama, A., Nakano, T. (2007). Micro X-ray CT at Spring-8 for Granular Mechanics. In Ling, H. I., Callisto, L., Leshchinsky, D., Koseki, J. (Eds.), Soil Stress-Strain Behavior: Measurement, Modeling and Analysis, 225-234. Springer Netherlands

Nakamura, A., Aizawa, K., (1984). Digital Circles. Computer Vision, Graphics, and Image Processing, 26, 2, 242-255

O'Gorman, L. (1996). Subpixel Precision of Staight-Edged Shapes for Registration Measurement. IEEE Transactions on Pattern Analysis and Machine Intelligence, 18, 7, 746-751

Pannier, Y., Lenoir, N., \& Bornert, M. (2010). Discrete volumetric digital image correlation for the investigation of granular type media at microscale: accuracy assessment. ICEM 14 - 14th International Conference on Experimental Mechanics. Poitiers: France.

Roscoe, K. H. (1970). The influence of strains in soil mechanics (10th Rankine Lecture). Géotechnique, 20, 2, 129-179.

Smit, R. C. A. (2010). Discrete 3D Digital Image Correlation (DIC) using particle reconnaissance. Masters Thesis, Delft University of Technology

Tejchman, J., Górski, J. (2010). Finite element study of patterns of shear zones in granular bodies during plane strain compression. Acta Geotechnica 5:95-112.

Vardoulakis, I., \& Sulem, J. (1995). Bifurcation Analysis in Geomaterials. London: Chapman \& Hall.

Wang, L. B., Frost, J. D., Lai, J. S. (2004). Three-Dimensional Digital Representation of Granular Material Microstructure from X-Ray Tomography Imaging. J. Comput. Civ. Eng., 18(1), 28-35.

Wang, L. B., Park, J. Y., Fu, Y. (2007). Representation of real particles for DEM simulation using X-ray tomography. Construction and Building Materials, 338-346.

Wang, Y. (2009). A new algorithm to model the dynamics of 3-D bonded rigid bodies with rotation. Acta Geotechnica 4:117-127. 